\title{
EFICÁCIA DOTESTE COMPUTADORIZADO DE ATENÇÃO VISUAL (TCA) E DO MINI EXAME DO ESTADO MENTAL (MEEM) NA IDENTIFICAÇÃO DE PORTADORES DE TRANSTORNO COGNITIVO LEVE
}

\author{
Fernanda Mayrink Gonçalves Zerbini \\ Universidade Castelo Branco, Rio de Janeiro, Brasil. \\ Maurício Rocha Calomeni \\ Universidade Castelo Branco, Rio de Janeiro, Brasil. \\ Vernon Furtado da Silva \\ Universidade Castelo Branco, Rio de Janeiro, Brasil.
}

Hebert Wilson dos Santos Cabral

Universidade Federal Fluminense, Niterói, Brasil.

Sérgio Luís Schmidt

Universidade Estadual do Rio de Janeiro, Brasil.

\begin{abstract}
Resumo
Com o envelhecimento populacional, as doenças da terceira idade têm recebido maior atenção. A Doença de Alzheimer é a forma mais comum de demência e o Transtorno Cognitivo Leve (TCL), período de transição entre as mudanças cognitivas do envelhecimento normal e o completo desenvolvimento das características clínicas de demência, tem sido amplamente estudado. O objetivo deste estudo foi avaliar 2 testes neuropsicológicos comparando sua acurácia diagnóstica na identificação de portadores de TCL. Os resultados demonstraram que os dois testes foram capazes de identificar os pacientes portadores de TCL, nenhum apresentou ser influenciado pelo efeito da idade, porém o Mini Exame do Estado Mental (MEEM) acusou influência da escolaridade.

Palavras-chave: Transtornos Mentais Orgânicos - Idoso Fragilizado - Diagnose - Memória.
\end{abstract}

\section{Introdução}

\begin{abstract}
A Doença de Alzheimer (DA) é a forma mais comum de demência, abrangendo 50-60\% dos casos (JOSHI, MORLEY, 2006; BLENNOW, DE LEON, ZETTERBERG, 2006) e acometendo aproximadamente 5\% dos adultos maiores de 65 anos (KLAFKI et al, 2006). Esse tipo de demência apresenta uma progressão lenta, com início insidioso, sendo que, os primeiros fatores a apresentarem deterioração são: a memória episódica e a capacidade atencional (BLENNOW, DE LEON, ZETTERBERG, 2006).

Estudos demonstram que a neurodegeneração e o transtorno em múltiplos domínios cognitivos estimativamente têm início de 20 a 30 anos antes do diagnóstico da doença (BLENNOW, DE LEON, ZETTERBERG, 2006; BACKMAN et al, 2004). Essa fase clínica é denominada Transtorno Cognitivo Leve (TCL) e se refere ao estado de transição entre as mudanças cognitivas do envelhecimento normal e o completo desenvolvimento das características clínicas de demência (LEVEY et al, 2006; 2007). Associado a uma análise da etiologia dos sintomas é possível presumir sobre o prognóstico do paciente e, em geral, o diagnóstico de TCL amnésico de uma etiologia possivelmente degenerativa, é mais provável de evoluir para DA (PETERSEN et al, 1999; GAUTHIER et al, 2006).
\end{abstract}


Visando a um diagnóstico precoce da DA, vários testes neuropsicológicos têm sido desenvolvidos. Com isso, o presente estudo enfocou dois desses testes, o Mini Exame do Estado Mental (MEEM) (FOLSTEIN; FOLSTEIN; MCHUGH, 1975) e o Teste Computadorizado de Atenção Visual (TCA) (SCHMIDT; MANHÃES, 2001), comparando a acurácia diagnóstica dos dois testes na identificação de portadores de TCL.

O Mini Exame do Estado Mental (MEEM) é mundialmente usado no diagnóstico do status mental. É um teste simples, de aplicação rápida (de 5 a 10 minutos), com pontuação que varia de 0 a 30 e suas questões avaliam: orientação temporal (5 pontos), orientação espacial (5 pontos), memória imediata (3 pontos), atenção e cálculo (5 pontos), memória de evocação (3 pontos), linguagem (8 pontos) e praxia visual e construtiva (1 ponto). Entretanto, deve-se ter cuidado ao avaliar a memória pelo MEEM, pois o esse exame é altamente influenciado pela idade e escolaridade, o que permite resultados "falso positivos" e "falso negativos" (BERTOLUCCI, 2000).

O TCA avalia a atenção visual central, impulsividade motora, tempo de reação visual e variabilidade do tempo de reação visual, por meio de quatro resultados quantitativos básicos: percentual de respostas omitidas - RO - (avalia atenção central a estímulos visuais); percentual de respostas incorretas - \%RI - (avalia impulsividade motora a estímulos visuais); tempo médio de reação aos estímulos visuais em milisegundos - TR - (avalia velocidade de reação motora a estímulos visuais); e variabilidade do tempo de reação visual em milisegundos - VR - (avalia capacidade de sustentar a atenção - concentração). O teste não dependente da linguagem e, principalmente, do efeito do aprendizado, sendo o tempo de aplicação do teste de 15 minutos (SCHMIDT, MANHÃES, 2001).

\section{Materiais e Métodos}

Os indivíduos que compuseram a amostra deste estudo foram selecionados em um centro de convivência privado na Serra - Espírito Santo, onde todos foram avaliados clinicamente por um médico e divididos em dois grupos. O grupo 1 foi composto pelos indivíduos idosos que acusaram queixas de memória (grupo TCL) e o grupo 2 foi composto pelos indivíduos idosos que não possuíam queixa de memória (grupo controle). Foram excluídos os indivíduos com idade inferior a 65 anos, portadores de déficit visual ou auditivo importante, que possuíam história de acidente vascular encefálico, doenças neurológicas degenerativas, depressão maior, delirium, história de traumatismo crânio-encefálico e diagnóstico prévio de demência.

Os indivíduos realizaram os dois testes (MEEM e TCA) e os dados obtidos foram analisados utilizando estatística descritiva, comparações de médias por Teste " $t$ " de Student, análises de variância ANOVA e MANOVA, cálculos de sensibilidade, especificidade, valores preditivos, falsos resultados, tendo como base a curva Receiver Operator Caracteristic Curve (ROC) e análises multivariadas por modelos de regressão logística. Foi utilizado pacote estatístico SPSS 14.0 por consultor contratado e fixado grau de significância em $\mathrm{p}<0,05$. Todos os indivíduos foram voluntários para a pesquisa, tendo-se de forma obrigatória o preenchimento do termo de consentimento livre e esclarecido, atendendo assim às normas para a realização de pesquisas com 
seres humanos, conforme a orientação do Conselho Nacional de Saúde, resolução 196/96, e aprovado pelo Comitê de Ética Institucional em Pesquisa da Universidade Castelo Branco - UCB/RJ.

\section{Resultados}

A análise descritiva das características sócio-demográficas dos grupos estudados contemplou os parâmetros: sexo, faixa etária e escolaridade. Não se observou diferença estatística concluindo que a amostra foi homogênea, como é mostrado na Tabela 1. Ainda nessa mesma tabela, na comparação dos escores avaliados do Mini Exame do Estado Mental (MEEM), os indivíduos TCL apresentaram uma média inferior quando comparados com os controles. Estes resultados são compatíveis com outros estudos como os de Petersen et al (1999) e Bertolucci (2000), que demonstram um pior desempenho no teste por pacientes com queixas cognitivas. A comparação do desempenho nos parâmetros, do Teste Computadorizado de Atenção Visual, também apresentada na Tabela 1 , apresentou diferença estatística no percentual de respostas incorretas (\%RI), que demonstra impulsividade, e na variabilidade do tempo de reação visual (VR), que reflete a concentração. Entretanto, não demonstrou diferença estatística nos parâmetros percentual de respostas omitidas (\%RO), que se relaciona à desatenção, e tempo de reação visual (TR), relacionado à velocidade de reação motora a estímulos visuais. O estudo de Cabral (2004) demonstra diferença significativa também no percentual de respostas omitidas.

Tabela 1: Descrição das características sócio-demográficas, comparação do mini exame do estado mental entre grupos, com índices de significância dados pelo teste quiquadrado e desempenho no teste computadorizado de atenção visual com índice de significância obtido através do teste $\mathrm{T}$ de student.

\begin{tabular}{|c|c|c|c|c|c|}
\hline \multicolumn{6}{|c|}{ Características Sócio-demográficas } \\
\hline \multirow[t]{2}{*}{ Parâmetro } & \multicolumn{2}{|c|}{$\begin{array}{c}\text { Controle } \\
(\mathrm{n}=23)\end{array}$} & \multicolumn{2}{|c|}{$\begin{array}{l}\text { TCL } \\
(n=38)\end{array}$} & \multirow{2}{*}{$\begin{array}{c}\text { Significância } \\
\text { teste* }^{*}\end{array}$} \\
\hline & No & $\%$ & No & $\%$ & \\
\hline \multicolumn{6}{|l|}{ Sexo } \\
\hline Masculino & 2 & 8,7 & 10 & 26,3 & \multirow{2}{*}{0,093} \\
\hline Feminino & 21 & 91,3 & 28 & 73,7 & \\
\hline \multicolumn{6}{|l|}{ Faixa etária } \\
\hline Até 70 anos & 13 & 56,5 & 16 & 42,1 & \multirow{2}{*}{0,275} \\
\hline 71 e mais anos & 10 & 43,5 & 22 & 57,9 & \\
\hline \multicolumn{6}{|l|}{ Escolaridade } \\
\hline 1 a 3 anos de estudo & 7 & 30,4 & 16 & 42,1 & \multirow{3}{*}{0,495} \\
\hline 4 a 7 anos de estudo & 12 & 52,2 & 14 & 36,8 & \\
\hline Mais de 7 anos de estudo & 4 & 17,4 & 8 & 21,1 & \\
\hline
\end{tabular}




\section{Comparação do Mini Exame do Estado Mental entre os grupos}

\begin{tabular}{c|c|c|c|c}
\multicolumn{2}{c|}{ Controle $(\mathrm{n}=23)$} & \multicolumn{2}{|c|}{ TCL $(\mathrm{n}=38)$} & \\
\cline { 1 - 3 } Média & DP & Média & DP & \multirow{2}{*}{0,001} \\
\hline 26,0 & 2,7 & 22,5 & 4,2 & \\
\hline
\end{tabular}

Comparação de desempenho no Teste Computadorizado de Atenção Visual

\begin{tabular}{c|c|c|c|c|c}
\hline \multirow{2}{*}{ Parâmetro } & \multicolumn{2}{|c|}{ Controle (n=23) } & \multicolumn{2}{|c|}{ TCL (n=38) } & $\begin{array}{c}\text { Significância } \\
\text { teste \# }\end{array}$ \\
\hline & Média & DP & Média & DP & \\
\hline \% RO & 2,42 & 7,45 & 4,57 & 5,76 & 0,212 \\
\hline \% RI & 4,12 & 4,47 & 7,78 & 4,60 & 0,004 \\
\hline TR & 434 & 51 & 455 & 66 & 0,212 \\
\hline VR & 93 & 30 & 138 & 63 & 0,002 \\
\hline
\end{tabular}

TCL = Transtorno Cognitivo Leve; DP= Desvio Padrão; TCL = Transtorno Cognitivo Leve; * Teste qui-quadrado; $\mathrm{DP}=$ Desvio Padrão; \# Teste t de Student; TCA (RO = Percentual de respostas omitidas); RI = Percentual de respostas incorretas; $\mathrm{TR}=$ Tempo médio de reação aos estímulos visuais; $\mathrm{VR}$ = Variabilidade do tempo de reação visual (milissegundos).

Já na Tabela 2 as análises de variância demonstraram a influência da escolaridade no resultado do MEEM, resultados já demonstrados nos estudos de Bertolucci (2000) e Canineu e Bastos (2002). Contudo, a mesma análise demonstrou não haver diferença significante nos parâmetros do TCA em relação à idade e à escolaridade, achado compatível com o estudo de Cabral (2004), demonstrando que este instrumento não sofre influência dos mesmos. Assim, utilizando as médias avaliadas pelo MEEM, identificou-se o melhor ponto de corte por meio da curva ROC para classificar os pacientes, como demonstrado na Figura 1, sendo este ponto encontrado de 24,5.

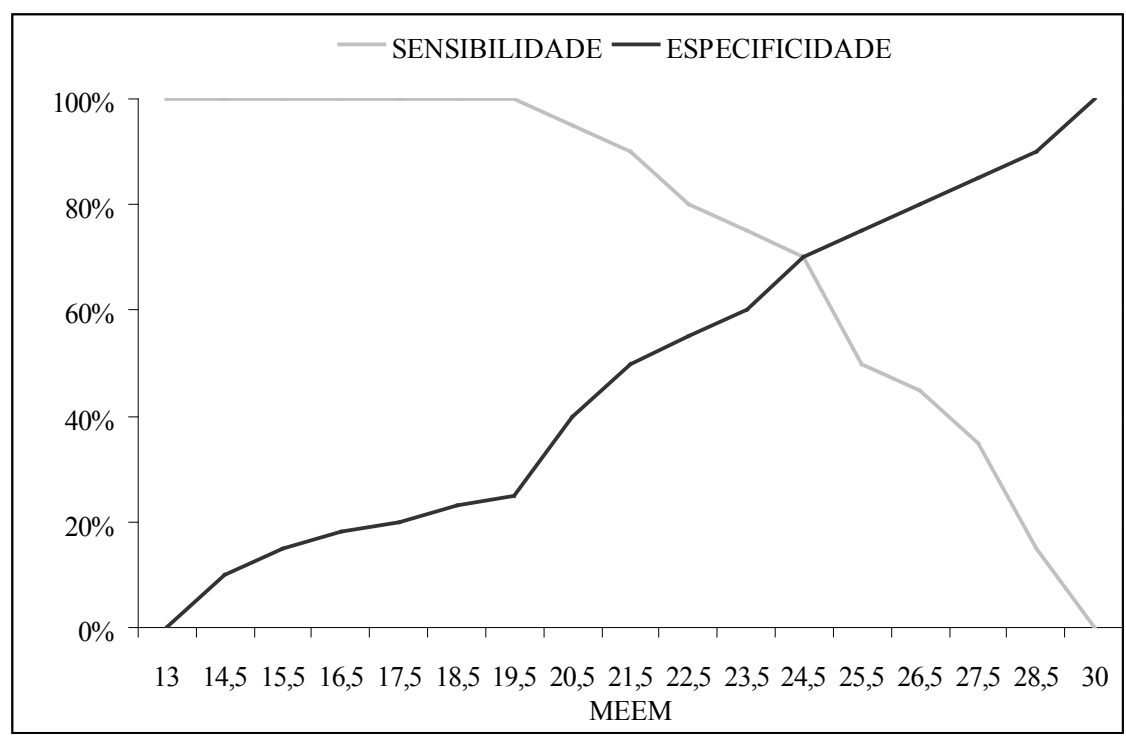

Figura 1: Apresentação gráfica da Curva ROC do Mini Exame do Estado Mental. 
A partir do corte foram calculados sensibilidade, especificidade, valor preditivo negativo, valor preditivo positivo, probabilidade de falsos positivos e probabilidade de falsos negativos, apresentados também na tabela 2 .

Tabela 2: Descrição da analise de Variância da idade, escolaridade e grupo no MEEM e TCA, com as classificações dos pacientes nos grupos pelo Mini Exame do Estado Mental e pelo Teste Computadorizado de Atenção, utilizando em ambos os casos o ponto de corte.

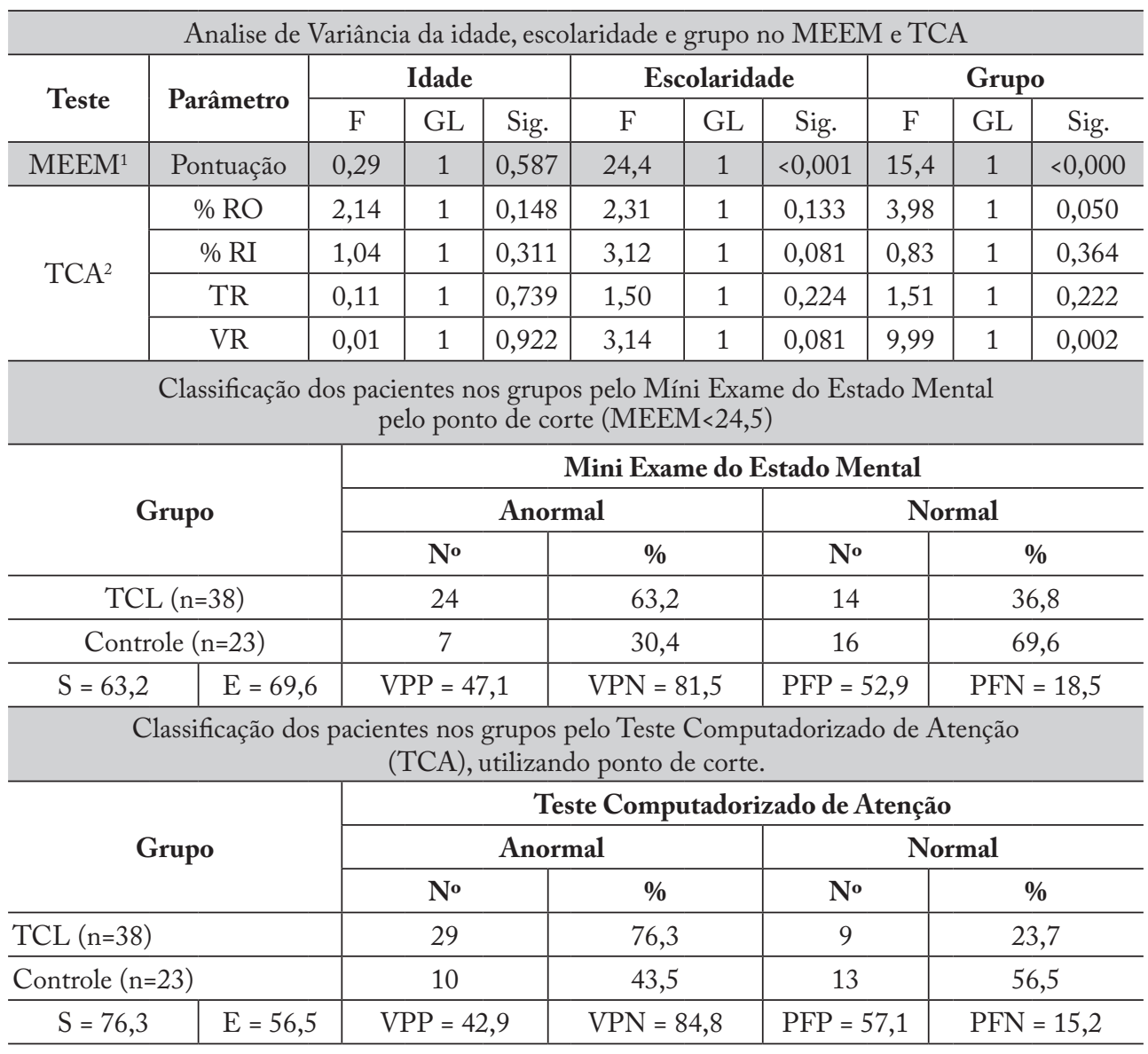

1 - Anova de 3 fatores (grupo, faixa etária e escolaridade); 2 - Manova (fator fixo=grupo; Covariáveis=idade e anos de escolaridade; TCA = Teste computadorizado de atenção; RO = Percentual de respostas omitidas; RI = Percentual de respostas incorretas; $\mathrm{TR}$ = Tempo médio de reação aos estímulos visuais; VR = Variabilidade do tempo de reação visual $(\mathrm{mm}) ;$ MEEM = Mini exame do estado mental; F= Fator; GL= Grau de liberdade; Sig = Significância; TCL $=$ Transtorno cognitivo leve $(\mathrm{S})=$ Sensibilidade $(\mathrm{E})$ = Especificidade $(\mathrm{VPP})$ = Valor Preditivo Positivo; $(\mathrm{VPN})=$ Valor Preditivo Negativo; $(\mathrm{PFP})$ = Probabilidade de Falsos Positivos; $(\mathrm{PFP})=$ Probabilidade de Falsos Negativos.

Da mesma forma que para o MEEM, utilizaram-se os parâmetros do TCA para determinar o ponto de corte, por meio da curva ROC. O melhor ponto de corte encontrado para o parâmetro omissão foi de 4,2, e, desta forma, também foi determinado o ponto de corte pela curva ROC para os demais parâmetros do TCA sendo erros de 0,9, tempo médio de reação (TR) de 441,0 e variabilidade do tempo de rea- 
ção (VR) de 103,5. A partir dos cortes foram calculados sensibilidade, especificidade, valor preditivo negativo, valor preditivo positivo, probabilidade de falsos positivos e probabilidade de falsos negativos, apresentados na Tabela 2. Para o TCA, foram considerados positivos para TCL pelo menos 2 parâmetros alterados.

Após a obtenção dos valores das especificidades, sensibilidades, fatores preditivos positivos e fatores preditivos negativos, foram calculadas as acurácias globais chegando aos resultados de 65\% para ambos os testes, como demonstrado no Figura 2.

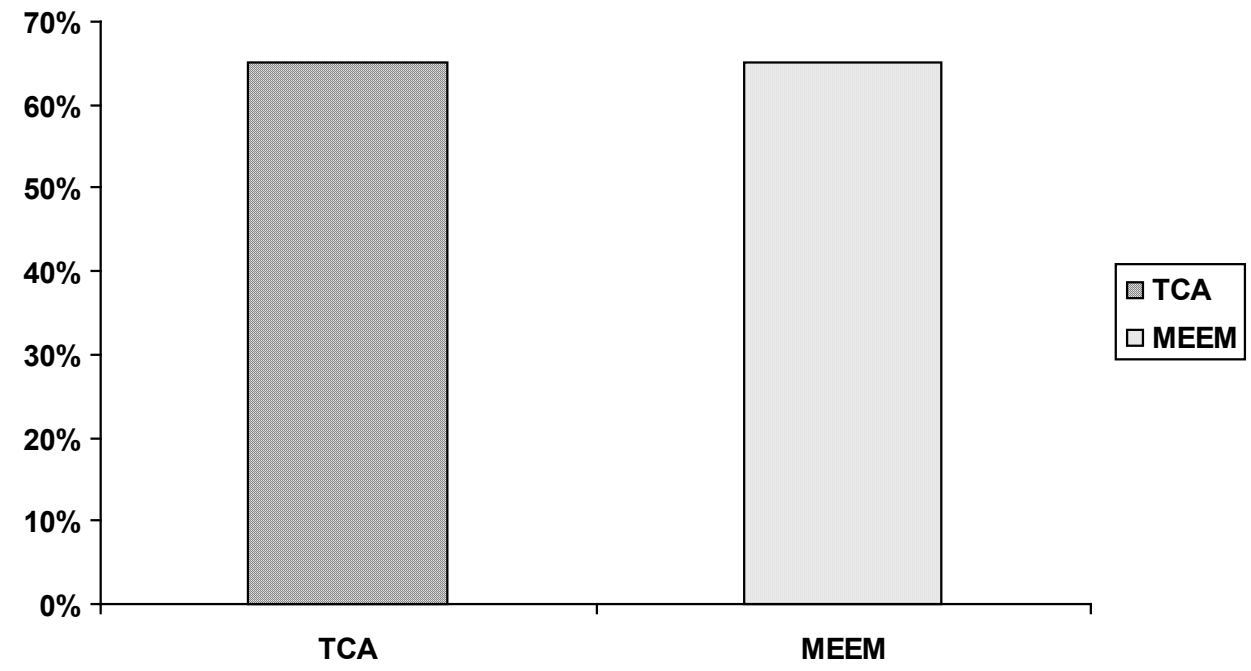

Figura 2: Índice de acurácia global calculado para o teste computadorizado de atenção (TCA) e para o Mini Exame do Estado Mental (MEEM) respectivamente.

O modelo multivariado por regressão logística identificou que, analisados separadamente os parâmetros do MEEM, o único capaz de predizer o TCL foi o parâmetro evocação, como demonstrado na Tabela 3.

Tabela 3: Apresentação dos resultados dos parâmetros, MEEM e TCA, avaliados na análise de regressão logística separadamente.

\begin{tabular}{l|c|c|c|c|c}
\hline \multicolumn{2}{c}{ Resultado dos parâmetros avaliados na análise de regressão logística } \\
\hline \multirow{2}{*}{ Parâmetro MEEM } & \multirow{2}{*}{$\mathrm{B}$} & \multirow{2}{*}{ Sig. } & \multirow{2}{*}{ Exp (B) } & \multicolumn{2}{c}{ IC 95\% } \\
\cline { 5 - 7 } & & & LI & LS \\
\hline Idade & $-0,071$ & 0,264 & 0,932 & 0,823 & 1,055 \\
\hline Anos de estudo & $-0,100$ & 0,350 & 0,904 & 0,733 & 1,116 \\
\hline MEEM - OT & 0,862 & 0,128 & 2,369 & 0,781 & 7,184 \\
\hline MEEM - OE & 1,428 & 0,061 & 4,171 & 0,939 & 18,532 \\
\hline MEEM - Memória imediata & 21,461 & 0,999 & $2 \mathrm{E}+009$ & 0,000 &. \\
\hline MEEM - Cálculo & $-0,153$ & 0,533 & 0,858 & 0,531 & 1,388 \\
\hline
\end{tabular}

(continua) 
(continuação)

\begin{tabular}{|c|c|c|c|c|c|}
\hline \multicolumn{6}{|c|}{ Resultado dos parâmetros avaliados na análise de regressão logística } \\
\hline \multirow{2}{*}{ Parâmetro MEEM } & \multirow{2}{*}{ B } & \multirow{2}{*}{ Sig. } & \multirow{2}{*}{$\operatorname{Exp}(B)$} & \multicolumn{2}{|c|}{ IC 95\% } \\
\hline & & & & LI & LS \\
\hline Idade & $-0,071$ & 0,264 & 0,932 & 0,823 & 1,055 \\
\hline Anos de estudo & $-0,100$ & 0,350 & 0,904 & 0,733 & 1,116 \\
\hline MEEM - Evocação & 0,773 & 0,046 & 2,167 & 1,014 & 4,630 \\
\hline MEEM - Linguagem & 0,967 & 0,074 & 2,630 & 0,909 & 7,611 \\
\hline MEEM - Praxia & $-0,087$ & 0,923 & 0,917 & 0,157 & 5,366 \\
\hline Constante & $-77,966$ & 0,999 & 0,000 & & \\
\hline \multicolumn{6}{|c|}{ Resultado dos parâmetros avaliados na análise de regressão logística } \\
\hline \multirow{2}{*}{ Parâmetro TCA } & \multirow{2}{*}{ B } & \multirow{2}{*}{ Sig. } & \multirow{2}{*}{$\operatorname{Exp}(B)$} & \multicolumn{2}{|c|}{ IC 95\% } \\
\hline & & & & LI & LS \\
\hline Idade & $-0,066$ & 0,261 & 0,936 & 0,835 & 1,050 \\
\hline Anos de estudo & $-0,002$ & 0,979 & 1,002 & 0,860 & 1,167 \\
\hline TCA - \%RI & 0,127 & 0,076 & 1,135 & 0,987 & 1,305 \\
\hline TCA - \%RO & $-0,095$ & 0,340 & 0,909 & 0,747 & 1,106 \\
\hline TR & 0,002 & 0,799 & 1,002 & 0,986 & 1,019 \\
\hline VR & $-0,044$ & 0,029 & 0,957 & 0,920 & 0,995 \\
\hline Constante & 7,917 & 0,088 & 2742,626 & & \\
\hline
\end{tabular}

MEEM= Mini exame do estado mental; OE= Orientação espacial; OT = Orientação temporal; B= Beta; Sig.= Significância; $\operatorname{Exp}(B)=$ Exponencial B; IC= Índice de confiança; LI= Limite inferior; LS= Limite superior; TCA= Teste computadorizado de atenção; $\mathrm{RO}=$ Percentual de respostas omitidas; $\mathrm{RI}=$ Percentual de respostas incorretas; $\mathrm{TR}=$ Tempo médio de reação aos estímulos visuais; $\mathrm{VR}$ = Variabilidade do tempo de reação visual ( $\mathrm{mm})$

O modelo multivariado por regressão logística identificou ainda que, analisados separadamente os parâmetros do TCA, o único capaz de predizer o TCL foi o parâmetro VR, como demonstrado na Tabela 3.

\section{Conclusão}

Os dados obtidos no presente trabalho demonstram que em ambos os instrumentos de rastreio, TCA e MEEM, os pacientes portadores de TCL apresentaram desempenho inferior ao grupo controle. Nenhum dos instrumentos apresentou efeito da idade, porém a escolaridade apresentou forte influência no MEEM, fato que não ocorreu no TCA.

Quando os parâmetros dos testes foram avaliados isoladamente, identificou-se que o que melhor prediz o TCL no MEEM são a evocação e a variabilidade do tempo de reação no TCA. Entretanto, na aplicação efetiva dos testes, os escores do MEEM não devem ser utilizados isoladamente, enquanto o TCA permite esta abordagem.

O instrumento TCA parece ter uma boa aplicabilidade na detecção do TCL e, corroborando para os achados, sua não influência pela escolaridade merece destaque, bem como sua objetividade e facilidade de aplicação. 
A amostra reduzida, o tipo de estudo descritivo e a necessidade de acompanhamento longitudinal, associados às perspectivas futuras do diagnóstico precoce da Doença de Alzheimer, demonstram a necessidade de maiores e intensos estudos sobre o tema e instrumentos de avaliação preditiva da doença.

The efficacy of the computerized visual attention test and of the mini-mental state examination (MMSE) in the identification of mild cognitive impairment (MCI) individuals

\begin{abstract}
Population aging has brought greater attention to old age diseases. Alzheimer's disease - the most common form of dementia, and mild cognitive impairment (MCI) - the transition period between the cognitive changes from regular aging and the full development of the clinical traits of dementia, have both been broadly studied. The aim of this study was to evaluate two neuropsychological tests, comparing their diagnosing accuracy in the identification of MCI individuals. Results have shown that both tests were capable of identifying MCI individuals with no influence from the age factor. MMSE, however, proved to be influenced by schooling.
\end{abstract}

Keywords: Organic Mental Impairment - Frail Elderly - Diagnosis - Memory.

Eficiencia de la atención visual computarizada test (ACT) y el miniexamen de estado mental (MMSE) en la identificación de las personas con deterioro cognitivo leve

Resumen

Con el envejecimiento de la población enfermedades de la vejez han recibido mayor atención. La enfermedad de Alzheimer es la forma más común de demencia y deterioro cognitivo leve (MCI), el período de transición entre los cambios cognitivos del envejecimiento normal y pleno desarrollo de las características clínicas de la demencia, ha sido ampliamente estudiado. El objetivo del estudio fue de 2 pruebas neuropsicológicas comparando su precisión diagnóstica en la identificación de las personas con MCI. Los resultados mostraron que ambas pruebas fueron capaces de identificar los pacientes con MCI, ninguno tuvo que ser influenciado por el efecto de la edad, sin embargo, el MMSE mostraron influencia de la escolarización. Palabras clave: Trastornos mentales orgánicos - Mayor debilitado - Diagnosticar - Memoria.

\title{
Referências
}

BACKMAN, L. et al. Multiple cognitive deficits during the transition to Alzheimer's disease. Journal of Internal Medicine, v. 256, p. 195-204, 2004.

BERTOLUCCI, P. H. F. Avaliação de memória. In: CARAMELLI, P.; FORLENZA, O. V. Neuropsiquiatria geriátrica. São Paulo: Atheneu, 2000, p. 507-516.

BLENNOW, K.; DE LEON, M.J.; ZETTERBERG, H. Alzheimer's disease. Lancet, v. 368, p. 387-403, 2006.

CABRAL, H. W. S. Transtorno cognitivo leve: contribuições para melhora da acurácia diagnóstica. 2004. 106 f. Dissertação (Mestrado em Neurologia) - Universidade Federal Fluminense, Niterói, 2004.

CANINEU, P. R.; BASTOS, A. Transtorno cognitivo leve. In: FREITAS, E. V. et al. Tratado de geriatria e gerontologia. Rio de Janeiro: Guanabara Koogan, 2002. cap. 14 , p. $128-132$. 
FOLSTEIN, M. F.; FOLSTEIN, S. E.; MCHUGH, P. R. Mini mental state: a pratical method for grading the cognitive state of patients for the clinician. J. Psychiatr Res., n.12, p. 198-198, 1975.

GAUTHIER, S. et al. Mild cognitive impairment. Lancet. v. 367, p. 1262-1270, 2006.

JOSHI, S.; MORLEY, J. E. Cognitive impairment. Med. Clin. North Am., v. 90, p. 769-787, 2006.

KLAFKI, H. W. et al. Therapeutic approaches to Alzheimer's disease. Brain. v. 129, p. 2840-2855, 2006.

LEVEY, A. et al. Mild cognitive impairment: na opportunity to identify patients at high risk for progression to Alzheimer's disease. Clin. Ther., v. 28, n. 7, p. 991-1001, 2006.

. Mild cognitive impairment: current research and clinical implications. Semin Neurol, New York, v. 27, p. 22-31, 2007.

PETERSEN, R. C. et al. Mild cognitive impairment clinical characterization and outcome. Arch. Neurol., v. 56, p. 303-308, 1999.

SCHMIDT, S. L.; MANHÃES, A.C. Teste computadorizado da atenção visual (TCA Vis). Rio de Janeiro: Cognição, 2001.

Recebido em: 09 de junho de 2009

Revisado em: 15 de junho de 2009

Aprovado em: 23 de junho de 2009

\section{Endereço para correspondência}

mauriciocalomeni@gmail.com

Mauricio Rocha Calomeni.

Universidade Castelo Branco/RJ -

Laboratório de Neuromotricidade e Performance Motora (LAMPEN).

Av. Salvador Allende, 6.700 - Recreio/RJ - CEP:22780-160 